DOI: https://doi.org/10.14311/TPFM.2021.011

\title{
INCREASING EJECTOR EFFICIENCY VIA DIFFUSER SHAPE OPTIMIZATION
}

\author{
Lucie Kubíčková ${ }^{1,2}$, Martin Isoz ${ }^{1,2}$, Jan Haidl ${ }^{3,4}$ \\ ${ }^{1}$ Czech Academy of Sciences, Institute of Thermomechanics, Dolejškova 5, Prague 18200 , \\ Czech Republic \\ ${ }^{2}$ Department of Mathematics, Faculty of Chemical Engineering, University of Chemistry and \\ Technology, Technická 5, Prague 166 28, Czech Republic \\ ${ }^{3}$ Czech Academy of Sciences, Institute of Hydrodynamics, Pod Patankou, Prague 166 12, \\ Czech Republic \\ ${ }^{4}$ Department of Chemical Engineering, Faculty of Chemical Engineering, University of Chem- \\ istry and Technology, Technická 5, Prague 166 28, Czech Republic
}

\begin{abstract}
An ejector is a technologically simple and yet wide-application fluid machine. While it has favorable characteristics for a significant number of technological processes, its main downside is probably its high operational energy demands. The present paper is an initial result of an ongoing research aimed at improving energy efficiency of the ejector via optimization of its geometry. In the paper, we focus mostly on presenting a general multi-objective optimization framework usable for an ejector shape optimization. The approach applicability is illustrated on a simplified problem comprising only a single phase flow in an ejector mixing tube and diffuser. Nevertheless, the achieved simulation and optimization results are validated against experimental data. The proposed optimization method itself is based on multi-objective evolutionary algorithms (MOEAs) combined with computational fluid dynamics (CFD) for evaluation of the vector-valued objective function.
\end{abstract}

Keywords: CFD, MOEA, ejector, OpenFOAM.

\section{Introduction}

Ejector pumps are technologically simple devices that utilize the kinetic energy of a high-velocity jet of a primary fluid to entrain the surrounding secondary fluid. Their simplicity and absence of any moving parts makes ejectors reliable for transport of dangerous or dirty fluids [1]. Furthermore, ejectors are used for example in refrigeration systems or in thermal systems for power generation [12]. Last but not least, liquid-gas ejectors have favorable mass transfer and mixing characteristics and as such they are used in chemical industry for applications in which the size of the gas-liquid interface is of a paramount importance [6].

A typical ejector unit comprises a nozzle, which transforms the primary (driving) fluid pressure into its kinetic energy, suction chamber for the entrance of the secondary (entrained) fluid, a converging part and mixing tube where the two fluids are mixed and the transport of momentum between the fluids takes place, and a diffuser for transforming the mixture kinetic energy back into the pressure, see Fig. 1-a).

The present work is a part of an ongoing research aimed at improving understanding and efficiency of ejectors. This initial study has two main objectives: (i) to develop a computational framework applicable to ejector shape optimizations, and (ii) to establish a workflow for validation of simulation and optimization results. As a first approximation of the ejector geometry, only the mixing tube and diffuser are taken into account, see Fig. 1-b). The goal of the study is to optimize the diffuser shape in order to increase energy efficiency of the simplified apparatus and to improve the quality of the device outlet stream. Note that after the simplification, the task at hand is similar to the work of Daniels et al. [3].

The sought optimization objectives are potentially conflicting and may be anti-correlated to a certain degree, i.e. it may not be achievable to improve a performance of a solution in one direction without deteriorating the performance in the other directions. As a robust tool to perform the outlined multi-objective optimization, we selected the evolutionary algorithms (EAs) first introduced by Schaffer [11]. 


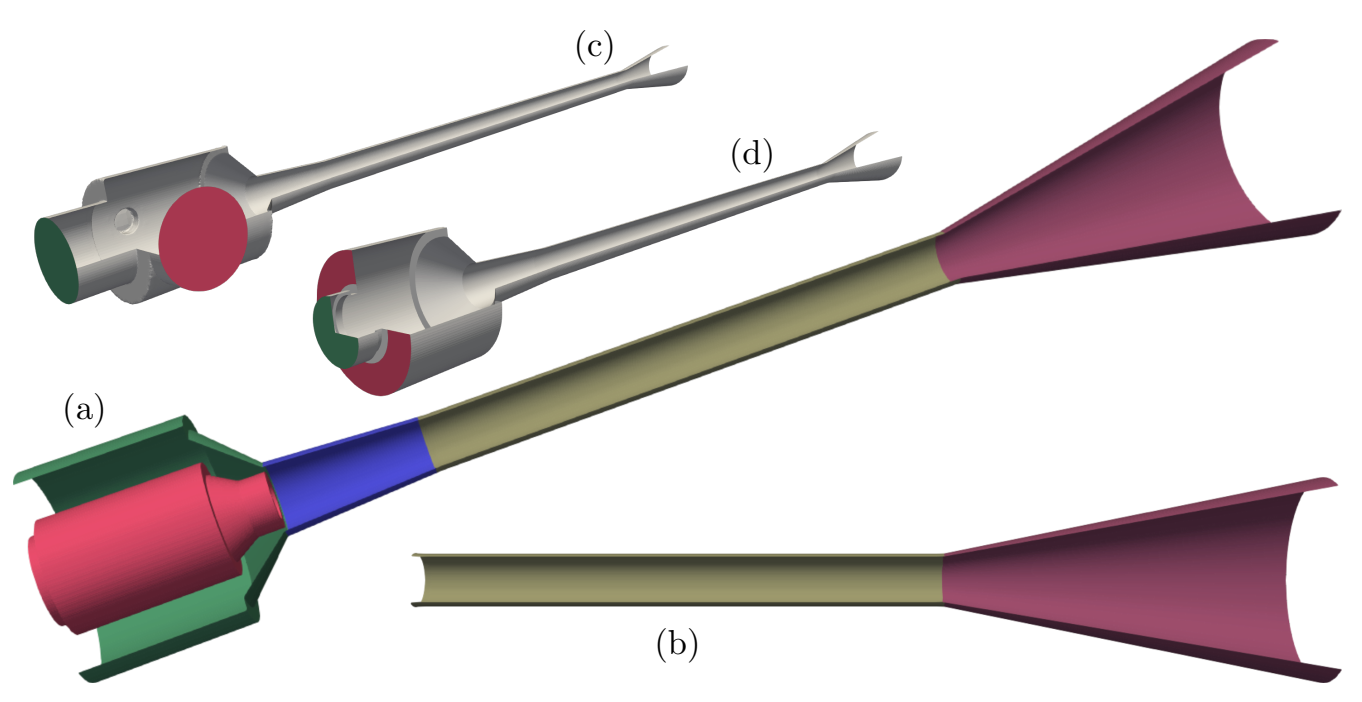

Figure 1: (a) Schematics of an axisymetric ejector comprising a nozzle (pink), suction chamber (green), converging part (blue), mixing tube (brown) and diffuser (violet). (b) Part of ejector of interest in the present work (c) three-dimensional model of the experimental ejector configuration with highlighted inlet (green) and suction (pink) (d) axi-symmetric approximation of (c).

\section{Experimental set-up}

The experimental validation was performed on a pilot scale upward pumping ejector. The detailed description of the set-up is given in [9]. For measurements relevant to this study, the nozzle was removed from the suction chamber and the inlet of the entrained fluid was closed. The measured section was mounted on the top of the suction chamber. The section consisted of the converging part (throat, $50 \mathrm{~mm}$ long), mixing tube of $18.0 \mathrm{~mm}$ i.d. divided into four individual parts (180 $\mathrm{mm}$, $90 \mathrm{~mm}, 45 \mathrm{~mm}, 65 \mathrm{~mm}$ ) and diffuser. The whole section was attached to the bottom of an $\approx 1.2 \mathrm{~m}$ high column of i.d. $300 \mathrm{~mm}$.

The experiments were conducted as static measurements of the pressure profiles along the setup. Tap water $\left(t=(18-22){ }^{\circ} \mathrm{C}\right)$ was pumped via a centrifugal pump while its flow rate was measured using an inductive flow meter (accuracy of $\pm 0.05 \mathrm{dm}^{3} \mathrm{~s}^{-1}$ ). Three water flow rates, $Q_{\mathrm{i}}^{\mathrm{o}}=\{1.0,2.0,2.9\} \mathrm{dm}^{3} \mathrm{~s}^{-1}$ were utilized. The pressures in the inlet pipe, in the suction chamber, along the mixing tube and at the bottom of the column were recorded (accuracy of $\pm 2 \mathrm{kPa}$ ).

Two diffuser designs were tested in this work: (i) default - a cone $130 \mathrm{~mm}$ long with the outlet i.d. of $50.0 \mathrm{~mm}$; (ii) optimized - see Fig. 2. The diffusers were 3D printed from ABS with $0.4 \mathrm{~mm}$ nozzle and $0.2 \mathrm{~mm}$ layer height. First $\approx 4 \mathrm{~cm}$ of the diffusers were brushed with a fine brush paper to remove the natural 3D print texture. Afterwards, the brushed walls were treated with an acetone solution to prepare a watertight surface without any sharp edges.

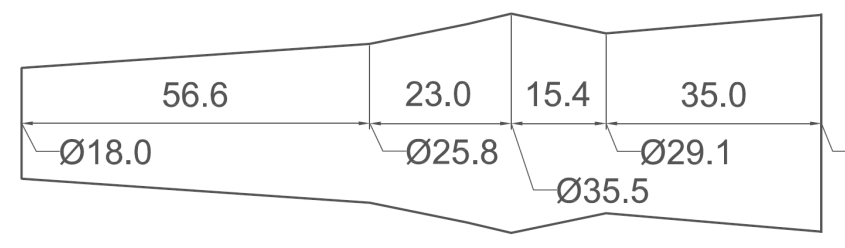

$\varnothing 35.2$

Figure 2: Sketch of the optimized diffuser geometry.

\section{Mathematical methods}

In order to computationally optimize the diffuser shape, a numerical model of the experimental apparatus was constructed in the open-source finite volume $\mathrm{C}++$ CFD library OpenFOAM [8]. 
The prepared CFD model was subsequently linked to the Platypus library [2], a free and opensource python library for multi-objective optimization. Using the CFD model for cost function evaluation in evolutionary algorithm requires the simulation to be fully automated, i.e. including the geometry generation and meshing, and fast to evaluate.

\subsection{Flow governing equations}

Model equations The flow in the experimental device was simulated as stationary isothermic and incompressible flow of a Newtonian fluid with the effects of turbulence taken into account via the Reynolds averaging. For the given case, the Reynolds averaged Navier-Stokes equations have the form of

$$
\begin{array}{rrr}
\nabla \cdot(\overline{\boldsymbol{u}} \otimes \overline{\boldsymbol{u}})-\nabla \cdot\left(\overline{\boldsymbol{\tau}}+\boldsymbol{\tau}^{\prime}\right) & = & -\nabla \bar{p}+\boldsymbol{g} \\
\nabla \cdot \overline{\boldsymbol{u}} & = & 0,
\end{array}
$$

where $\overline{\boldsymbol{u}}$ and $\bar{p}$ are the averaged velocity and kinematic pressure, respectively. Next, $\overline{\boldsymbol{\tau}}=\nu \nabla \overline{\boldsymbol{u}}$ is the averaged viscous stress tensor and $\nu$ marks the fluid kinematic viscosity. The second stress tensor, $\boldsymbol{\tau}^{\prime}=\boldsymbol{u}^{\prime} \otimes \boldsymbol{u}^{\prime}$ corresponds to the so-called Reynolds stress tensor, and $\boldsymbol{u}^{\prime}$ is the instantaneous turbulence-driven velocity fluctuation. Finally, $\boldsymbol{g}$ is a body force representing the gravity. Note that hereafter, only Reynolds averaged variables will be taken into account and the bar over individual symbols will be omitted.

Due to the presence of the Reynolds stress term $\nabla \cdot \boldsymbol{\tau}^{\prime}$, the formulation (1) requires additional closure model. There are numerous RANS closure models available in both literature and OpenFOAM. Due to the expected adverse pressure gradient in the diffuser and in agreement with Daniels et al. [3], we selected the Menter's $k-\omega$ shear stress transport (SST) model in the formulation given by Hellsten [7]. Discretization schemes were selected with a strong focus on the model robustness. The pressure and velocity discretizations were predominantly of second order. However, strong flux limiters resulting in mostly first order discretization were used for turbulence variables.

\subsection{Problem geometry and boundary conditions}

Three different model geometries were tested, (i) fully three-dimensional digital twin of the experimental device (Fig. 1-c)), (ii) axi-symmetric approximation of the experimental device (Fig. 1-d)), and (iii) axi-symmetric approximation of the mixing tube and diffuser (Fig. 1-b)).

The axial symmetry of the real-life ejector is disturbed by the presence of the secondary fluid inlet, c.f. Fig. 1-a) and c). Under standard configuration, the nozzle produces a high velocity jet that pumps the fluid from the secondary inlet. However, during the experiments, no nozzle was installed in the suction chamber and the secondary inlet was blind. Due to the device modified geometry, large vortices were formed in the suction chamber resulting to an asymmetric averaged velocity and pressure distribution at the throat inlet and potentially affecting the profiles in the part of the interest. In the axi-symmetric approximations of the experimental device, both primary

\begin{tabular}{|c|c|c|c|}
\hline Boundary & Conditions & Boundary & Conditions \\
\hline$\partial \Omega_{\text {inlet }}$ & $\begin{array}{c}\boldsymbol{u}=-Q_{\mathrm{i}} \boldsymbol{n}_{\mathrm{i}} / \text { Area }_{\text {inlet }} \\
\boldsymbol{n}_{\text {inlet }} \cdot \nabla p=0 \\
k=k_{\mathrm{i}}, \omega=\omega_{\mathrm{i}}\end{array}$ & $\partial \Omega_{\text {wall }}$ & $\begin{array}{c}\boldsymbol{u}=(0,0,0)^{\mathrm{T}} \\
\mathbf{n}_{\text {wall }} \cdot \nabla p=0 \\
k, \omega \leftarrow \text { wall function }\end{array}$ \\
\hline$\partial \Omega_{\text {outlet }}$ & $\begin{array}{c}\boldsymbol{n}_{\text {outlet }} \cdot \nabla \boldsymbol{u}=(0,0,0)^{\mathrm{T}} \\
p=0 \\
\boldsymbol{n}_{\text {outlet }} \cdot \nabla k=\boldsymbol{n}_{\text {outlet }} \nabla \omega=0\end{array}$ & & \\
\hline
\end{tabular}
and secondary inlets are aligned, see Fig. 1-d).

Table 1: Applied boundary conditions, by $Q$ we mark the volumetric flow rate and $\boldsymbol{n}$ denotes outer unit normal to the boundary. The inlet value of turbulence variables are estimated to correspond to $4 \%$ turbulence intensity and length scale equal to the mixing tube i.d. (18 mm).

The complete list of prescribed boundary conditions for all the variables of interest is given in Tab 1. For $\Omega \subset \mathbb{R}^{3}$ representing an open simply-connected computational domain corresponding to 
the ejector internals, the boundary of $\Omega$ is denoted $\partial \Omega$ and divided into three different subdomains,

$$
\partial \Omega=\partial \Omega_{\text {inlet }} \cup \partial \Omega_{\text {outlet }} \cup \partial \Omega_{\text {wall }},
$$

where $\partial \Omega_{\text {inlet }}$ represents the primary fluid inlet, $\partial \Omega_{\text {outlet }}$ is the outlet from the ejector and $\partial \Omega_{\text {wall }}$ represents the system solid boundaries.

\subsection{Multi-objective optimization with evolutionary algorithms}

Diffuser performance indicators The main purpose of a single-phase ejector is to use the momentum of the driving primary fluid to induce motion of the secondary, or entrained, fluid. The primary energy and momentum source in the ejector is the pressure overhead at $\partial \Omega_{\text {inlet }}$. This pressure energy is subsequently converted into kinetic energy of both the primary and secondary fluid and finally it is converted back to the pressure energy in the diffuser.

The efficiency of the pressure recovery in the diffuser was evaluated via the so-called pressure recovery factor $c_{p}$. The pressure recovery factor is defined in agreement with [3] as

$$
c_{p}=\frac{1}{\frac{1}{2} \rho u_{\mathrm{ref}}}\left[\frac{1}{\left\|A_{1}\right\|} \int_{A_{1}} p d S-\frac{1}{\left\|A_{\mathrm{ref}}\right\|} \int_{A_{\mathrm{ref}}} p d S\right],
$$

where $p$ is the static pressure, $\rho$ is the fluid density and $u_{\text {ref }}$ is the mean streamwise velocity over $A_{\text {ref }}$. For the purpose of this investigation, we selected $A_{1} \equiv \partial \Omega_{\text {outlet }}$ and placed $A_{\text {ref }}$ one centimeter before the end of the mixing tube.

The second used performance indicator is selected based on an assumption that an ejector may be equipped as a part of a production line. Therefore, it is desirable to maintain uniformity of the outflow from the ejector. The outflow uniformity was quantified via the flow uniformity index $\gamma$ introduced by Weltens et al. [13] and defined as

$$
\gamma=1-\frac{1}{2}\langle\omega\rangle_{\mathrm{avg}}^{A}, \quad \omega=\left|u_{\perp}^{A}-\left\langle u_{\perp}^{A}\right\rangle_{\mathrm{avg}}^{A}\right|, u_{\perp}^{A}=\boldsymbol{n}_{A} \cdot \boldsymbol{u},\langle x\rangle_{\mathrm{avg}}^{A}=\frac{1}{\|A\|} \int_{A} x d S,
$$

where $\boldsymbol{n}_{A}$ is a outer unit normal field to the surface $A$. In the following, we put $A:=\partial \Omega_{\text {outlet }}$ and orient the plane $A$ in a way that $\boldsymbol{n}_{A}$ is parallel to the main flow direction, i.e. to the ejector axis of symmetry.

Using the relation (4), $\gamma \in[0,1]$, where $\gamma=0$ is the worst case, $\gamma=1$ the ideal case and $\gamma=0.5$ corresponds for example to the case where $u_{\perp}^{A}=0$ at half of the control plane $A$ while the remaining half of $A$ experiences double the average velocity [13].

Note that the pressure recovery factor $\left(c_{p}\right)$ is closely related to the ejector energy efficiency. Furthermore, the two selected performance indicators are expected to show some degree of correlation between themselves. In particular, as stated by Daniels et al. [3], the increasing uniformity at the ejector outflow should aid the pressure recovery. Nevertheless, the exact form of correlation between $c_{p}$ and $\gamma$ is unclear and the two objectives need to be assumed possibly anti-correlated.

Multi-objective optimization problem We aim to optimize the diffuser shape by maximizing two possibly anti-correlated objectives. In general, let $\mathcal{P} \subset \mathbb{R}^{n}$ be a parameter space, $\mathcal{S} \subset \mathbb{R}^{m}$ be an objective space and $\boldsymbol{f}: \mathcal{P} \rightarrow \mathcal{S}$ be a vector-valued cost function. In our particular case, $m=2$ and the multi-objective optimization problem (MOP) can be written as

$$
\min _{\boldsymbol{p} \in \mathcal{P}} f_{1}(\boldsymbol{p})=-\frac{1}{N} \sum_{i=1}^{N} c_{p, i}, \quad \min _{\boldsymbol{p} \in \mathcal{P}} f_{2}(\boldsymbol{p})=-\frac{1}{N} \sum_{i=1}^{N} \gamma_{i},
$$

where $\boldsymbol{p}$ is a vector defining the diffuser shape and the terms on the right hand side, $c_{p}$ and $\gamma$, are pressure recovery factor and flow uniformity index, respectively. Finally, $N$ is the number of flow rates tested for a single geometry.

The conflicting nature of the objectives precludes the existence of an ideal solution because improving performance in one direction may deteriorate the performance in the other one. Thus, we aim to find a set of trade-off solutions where the best set possible is called a Pareto set and its projection into $\mathcal{S}$ the Pareto front, see [10] or Def. 1. 
Definition 1 (Pareto dominance, Pareto optimal set and Pareto optimal front) Let $\boldsymbol{p}_{1}, \boldsymbol{p}_{2} \in \mathcal{P}$. The solution $\boldsymbol{p}_{1}$ is said to dominate $\boldsymbol{p}_{2}\left(\boldsymbol{p}_{1} \succ \boldsymbol{p}_{2}\right)$ if and only if $\forall i \in 1 \ldots m: f_{i}\left(\boldsymbol{p}_{1}\right) \leq f_{i}\left(\boldsymbol{p}_{2}\right)$ and $\exists j \in 1 \ldots m: f_{j}\left(\boldsymbol{p}_{1}\right)<f_{j}\left(\boldsymbol{p}_{2}\right)$. For a given $M O P$, let $P^{*}$ denote all $\boldsymbol{p} \in \mathcal{P}$ such that $\nexists \boldsymbol{p}^{\prime} \in \mathcal{P}: \boldsymbol{p}^{\prime} \succ \boldsymbol{p}$. Let the set $P^{*}$ be called the Pareto optimal set and the set $\boldsymbol{f}\left(P^{*}\right)$ the Pareto optimal front.

However, finding the exact Pareto set may be impossible in a finite time. Therefore, a sufficiently good approximation $\hat{P}^{*}$ of the Pareto set $P^{*}$ is accepted as a feasible optimization result. Ultimately, the multi-objective optimization problem becomes a task of computing $\hat{P}^{*}$.

For the used axi-symmetric approximation, the vector variable $\boldsymbol{p}$ in (5) comprises radial and axial coordinates of four control points and the width of the diffuser outlet, $\boldsymbol{p} \in \mathcal{P} \subset \mathbb{R}^{9}$. The final diffuser shape was obtained by linear interpolation. The interpolation points comprised the fixed end of the mixing tube, the four control points and the diffuser end point with fixed axial and optimized radial coordinate. A piece-wise linear design was selected with the hope to discard the solutions dependent on an exact position of the separation point on a smooth edge from the Pareto optimal set and to increase the robustness of the identified optimal designs.

Multi-objective Evolutionary Algorithms The problem (5) was solved within the framework of multi-objective evolutionary algorithms (MOEAs). The advantage of such an approach is that it treats the cost function as a black box and does not make any assumptions on its properties [5]. As such, MOEAs are very robust and suitable for optimizations based on CFD-evaluated cost functions [3]. The results presented in this work were computed using NSGA-II algorithm of Deb et al. [4]. For any tested geometry, the cost function in (5) is evaluated from CFD simulation of flow in the axi-symmetric approximation of the mixing tube and diffuser for three different fluid flow rates of $Q_{\mathrm{i}}=\{1.5,3.0,4.5\} \mathrm{dm}^{3} \mathrm{~s}^{-1}$.

\section{Results}

\subsection{Suitable mesh size and model validation}

Mesh resolution Because the part of utmost interest in the present study is the diffuser, the resolution of the computational mesh was determined based on the axi-symmetric simulation of the mixing tube and the diffuser. Meshes with an approximate cell scale ranging from 0.25 to 1.0 $\mathrm{mm}$ were tested for three different flow rates of $Q_{\mathrm{i}}^{\mathrm{o}}=\{1.5,3.0,4.5\} \mathrm{dm}^{3} \mathrm{~s}^{-1}$. The selected flow rates cover the whole range of experimental data and are the same as used for the cost function evaluation in MOEA.

All the meshes used in axi-symmetric computations were structured and refined towards the walls. The intensity of mesh refinement towards the walls changed with the flow Reynolds number. The goal of such a treatment is to always have the three flow rates computed with similar values of $y^{+}$at the walls.

The results of mesh size independence study are given in Fig. 3. The pressure recovery factor $\left(c_{\mathrm{p}}\right)$ stayed roughly the same for all the tested meshes and fluid flow rates. The mesh uniformity index $(\gamma)$ decreases with increasing mesh resolution. At first, the low mesh resolution adds a substantial numerical diffusion of the flow core that artificially improves $\gamma$. Afterwards, increasing the mesh resolution limits this numerical diffusion and first mesh-independent solution for all flow rates is obtained for cell scales roughly between 0.5 and $0.7 \mathrm{~mm}$.

Increasing the mesh resolution further causes $y^{+}$at the mixing tube-diffuser transition to fall below 30 and $\gamma$ starts to change again. A second mesh-independent solution may be expected for a fully resolved boundary layer $\left(y^{+} \lesssim 1\right)$. However, such a mesh resolution would lead to simulation times unusable for MOEAs-based optimizations. Consequently, the mesh resolution corresponding to the cell scale $\simeq 0.5 \mathrm{~mm}$ was selected for all the subsequent computations and all the three model geometries.

Model validation To validate the prepared CFD models, we measured pressure profiles along the ejector operated with prescribed fluid flow rates at inlet and a closed-of suction. Results of the three models as well as the experimental data for three selected flow rates are shown in Fig. 4. The agreement between experiment and simulation is not perfect, especially in the middle part 


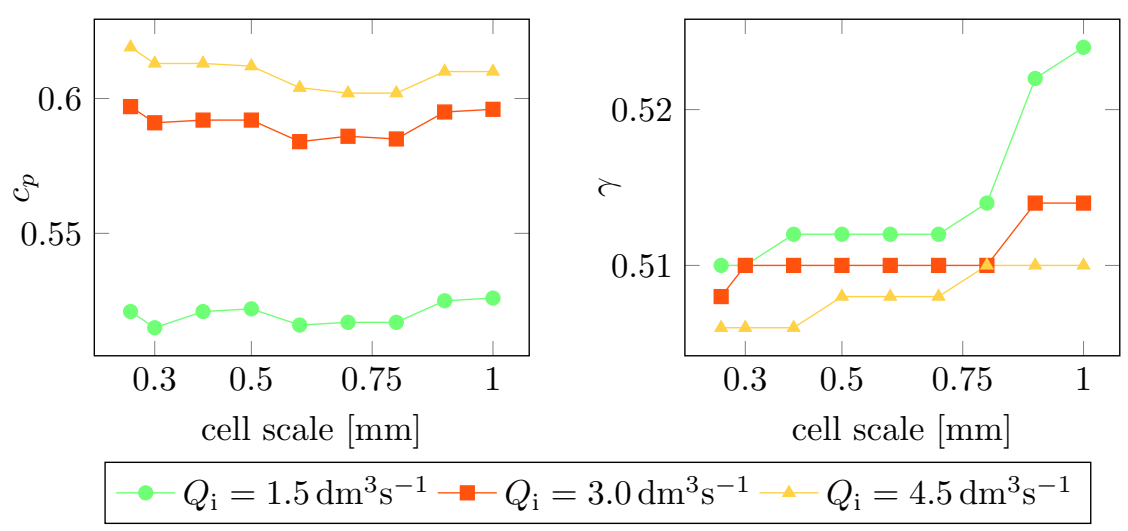

$1.00 \mathrm{~mm}$ (coarse)

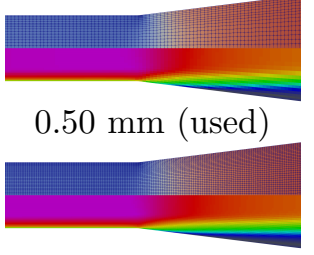

$0.25 \mathrm{~mm}$ (fine)

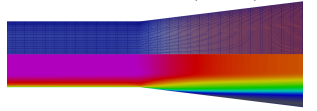

Figure 3: Dependence of the diffuser performance indicators on the mesh resolution for axisymmetric simulation of flow inside the mixing tube and diffuser. Qualitative view of the coarsest, used and finest tested meshes is given on the right. The qualitative views are focused on the transition between the mixing tube and the diffuser. Each view contains mesh structure and pressure field (top) and velocity field (bottom).

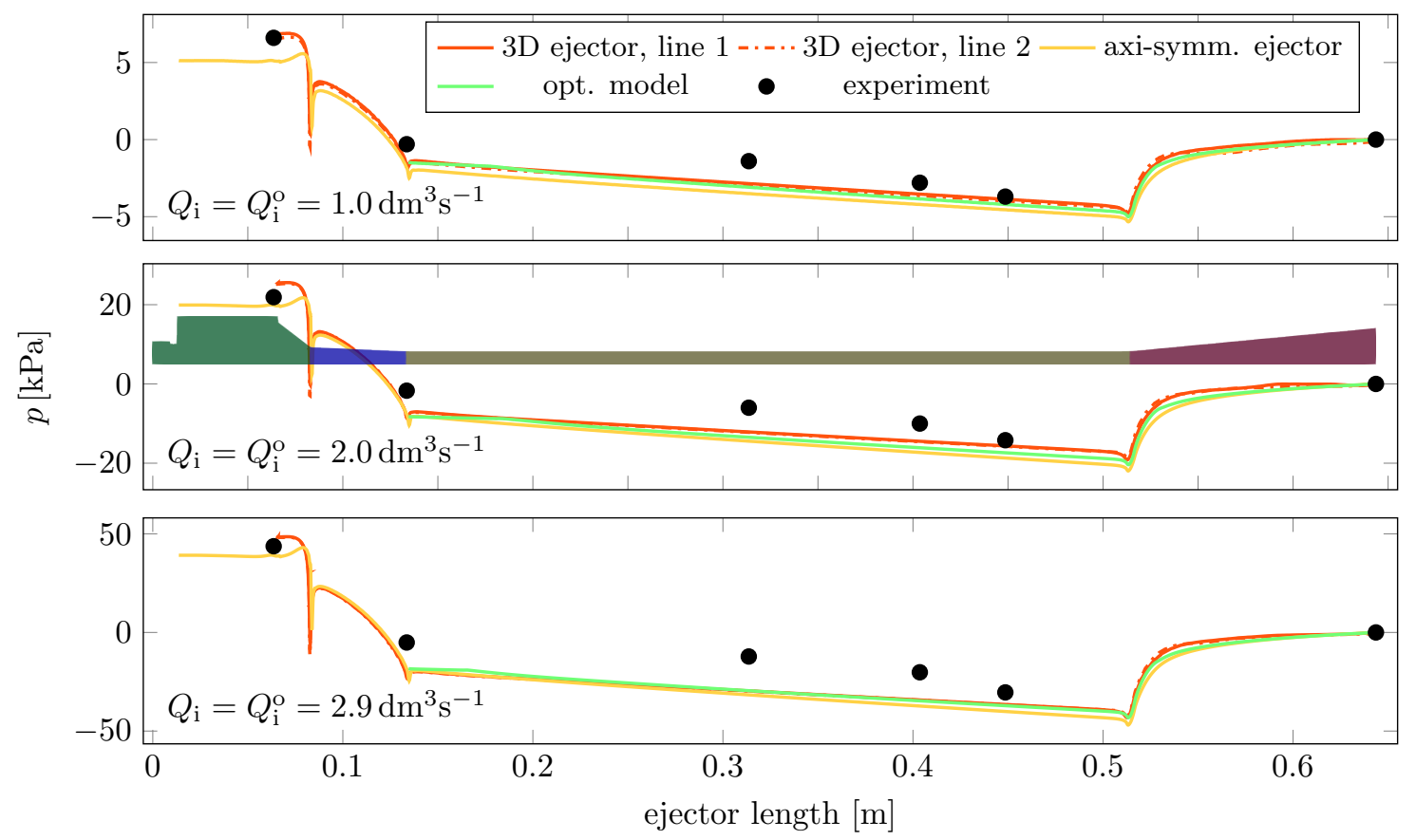

Figure 4: Pressure profiles along the ejector wall for the three model geometries and experiment. The 2D computational domain is added for better connectivity. 
of the mixing tube. However, the fully $3 \mathrm{D}$ simulation is capable to predict the pressure in the suction chamber quite accurately. Furthermore, the difference between the last pressure measured inside the mixing tube and the pressure at the diffuser outlet seems to be picked-up by the model with acceptable accuracy. Finally, no qualitative differences in the pressure profiles were observed for the three models, especially with respect to the mixing tube and the diffuser. Consequently, the qualitative trends in optimal diffuser shape resulting from MOEA optimization based on the simplified axi-symmetric model are expected to hold for more complex models and for the real-life device.

\subsection{Optimization results}

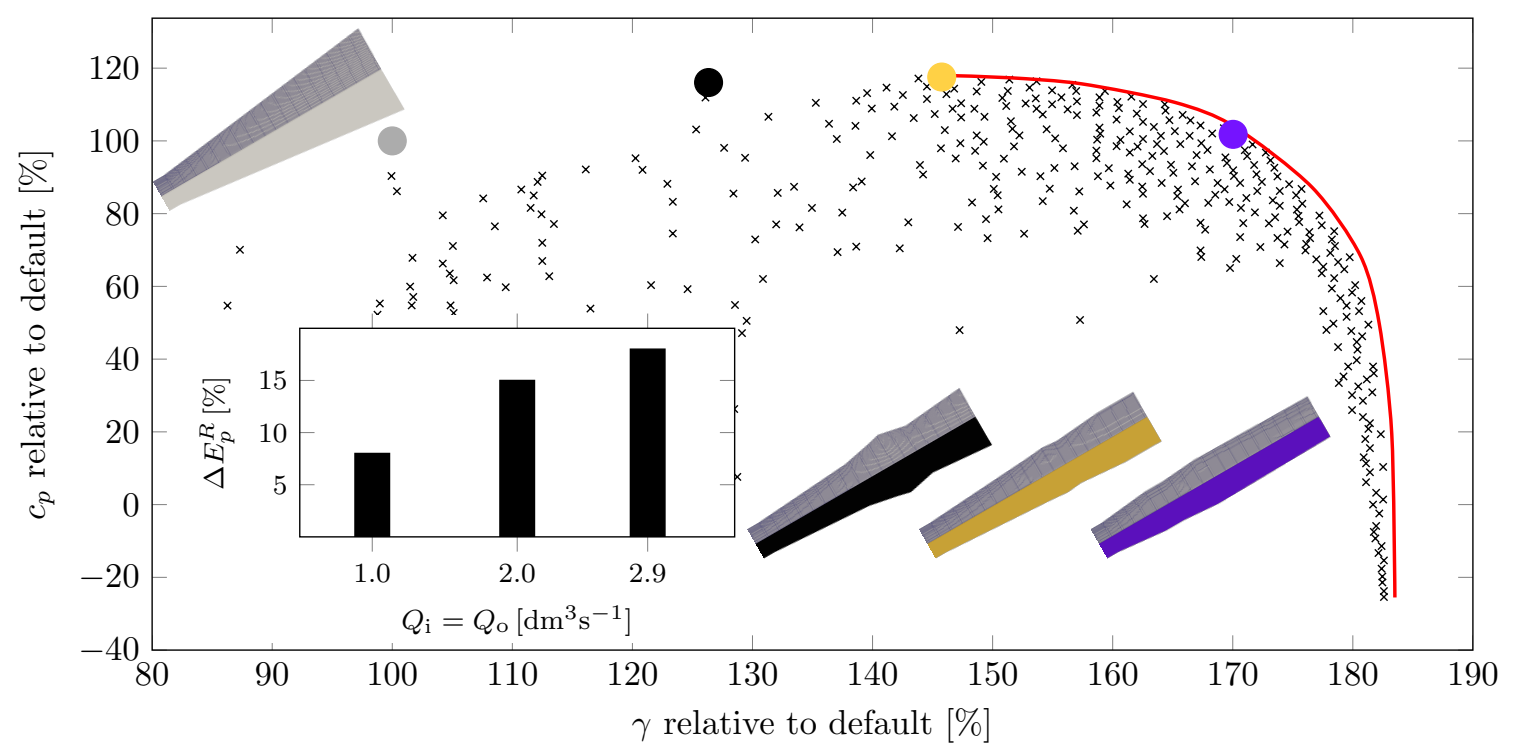

Figure 5: Results of multi-objective optimization. All the depicted example geometries are in the same scale. Each black cross corresponds to a CFD simulation. Pareto front is indicated in red. The default geometry is gray. An experimentally measured change in the ejector pressure energy efficiency using the black diffuser is given in the subplot.

The MOEA optimization using NSGA-II algorithm [4] with 480 individuals in a population and 20 generations yielded the results depicted in Fig. 5. The most energy-efficient design identified by the optimization corresponds to the yellow point in Fig. 5. However, as mentioned in the description of the experiment, the diffusers had to be 3D-printed to allow testing arbitrary shapes resulting from the optimization. Consequently, there was a fine texture present in the internals of all the tested diffusers. Based on additional computational analysis, we estimated that the texture-caused performance loss of the most energy-efficient diffuser design is rather high. In particular, approximately 1.5 higher than the performance loss of a sub-optimal yet robust design depicted in black in Fig. 5, which was selected for experimental validation. Finally, note that all the pareto-optimal designs have a significantly better quality of output stream than the default configuration.

In the subplot of Fig. 5, we show the measured relative changes in ejector pressure energy efficiency defined as $\Delta E_{p}^{R}=\left(\Delta p_{\mathrm{b}}-\Delta p_{\mathrm{o}}\right) / \Delta p_{\mathrm{b}} \cdot 100[\%]$, where $\Delta p$ is the pressure difference between the ejector inlet and outlet and the subscripts "b" and "o" mark the baseline and optimized geometry, respectively. The average measured improvement in ejector pressure energy efficiency for the three tested flow rates was approximately 13\%. Furthermore, the new design seems to behave better at higher fluid flow rate, which is closer to the real-life ejector operation conditions.

\section{Conclusion}

In the present contribution, we outlined an optimization framework based on coupling computational fluid dynamics with multi-objective evolutionary algorithms. The framework was applied to 
the shape optimization of a diffuser attached to an ejector. The main optimization goals were to increase the ejector energy-efficiency while simultaneously improve its outlet stream quality. Both the CFD model used for optimization and the optimization results were validated using specifically designed experiment. The diffuser shape selected based on the performed optimization showed approximately $13 \%$ improvement in pressure energy efficiency compared to the baseline design. In the subsequent work, the framework will be applied to a single-phase ejector operated under standard conditions and the optimization objective will be extended by maximization of the ratio between mass flow rates of the entrained and the driving fluids.

\section{Acknowledgment}

The work was supported by the Grant project with No. GC19-02288J of the Czech Science Foundation, within institutional support RVO:61388998 and by the Centre of Excellence for Nonlinear Dynamic Behaviour of Advanced Materials in Engineering CZ.02.1.01/0.0/0.0/15_003/0000493 (Excellent Research Teams) in the framework of Operational Programme Research, Development and Education.

\section{References}

[1] Choi, S., Ji, H. \& Kim, K.: Comparative study of hydrodynamic characteristics with respect to direction of installation of gas-liquid ejector system. J. Mech. Sc. Technol. vol. 29 (2015). pp. $3267-3276$.

[2] D. Hadka. Platypus, A: Free and Open Source Python Library for Multiobjective Optimization (2015). URL https://github.com/Project-Platypus/Platypus.

[3] Daniels, S., Rahat, A., Tabor, G., Fieldsend, J. \& Everson, R.: Automated shape optimization of a plane asymmetric diffuser using combined Computational Fluid Dynamic simulations and multi-objective Bayesian methodology. Int. J. Comp. Fl. Dyn. (2019). pp. 1683165-1$1683165-17$.

[4] Deb, K., Pratap, A., Agarwal, S. \& Meyarivan, T.: A fast and elitist multiobjective genetic algorithm: NSGA-II. IEEE Transs on Evol. Comput. vol. 6 no. 2 (2002). pp. 182-197.

[5] Emmerich, M. \& Deutz, A.: A tutorial on Multiobjective Optimization: Fundamentals and Evolutionary Methods. Nat. Comput. vol. 17 (2018). pp. 585-609.

[6] Havelka, P., Linek, V., Sinkule, J., Zahradnik, J. \& Fialova, M.: Effect of the ejector configuration on the gas suction rate and gas hold-up in ejector loop reactors. Chem. Eng. Sci. vol. 52 (1997). pp. 1701-1713.

[7] Hellsten, A.: Some improvements in Menter's k- $\omega$ SST turbulence model. In Proceedings of the Fluid Dynamics Conference: volume 71: page 11. AIAA (1997).

[8] OpenCFD: OpenFOAM: The Open Source CFD Toolbox. User Guide Version 1.4, OpenCFD Limited. Reading UK (2007).

[9] Opletal, M., Novotny, P., Linek, V., Moucha, T. \& Kordac, M.: Gas suction and mass transfer in gas-liquid up-flow ejector loop reactors. effect of nozzle and ejector geometry. Chem. Eng. J. vol. 353 (2018). pp. 436-452.

[10] Pareto, V.: Course d'Economie Politique. Librairie Droz (1896).

[11] Schaffer, J.: Multiple objective optimization with vector evaluated genetic algorithms. In Grefensette, G. \& Erlbraum, J. L., editors, Proceedings of the First Int. Conference on Genetic Algortihms: pp. 93-100 (1985).

[12] Taleghani, S., Sorin, M. \& Poncet, S.: Modeling of two-phase transcritical $\mathrm{co}_{2}$ ejectors for on-design and off-design conditions. Int. J. Refr. vol. 87 (2018). pp. 91-105.

[13] Weltens, H., Bressler, H., Terres, F., Neumaier, H. \& Rammoser, D.: Optimisation of catalytic converter gas flow distribution by CFD prediction. SAE Tech. Pap. Series. vol. 930780 (1993). pp. $1-22$. 